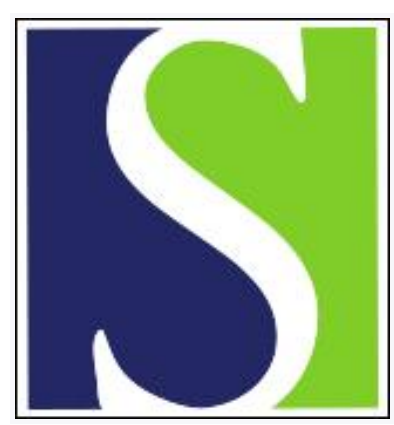

Scand J Work Environ Health 1992;18(6):410

https://doi.org/10.5271/sjweh.1554

Issue date: 01 Dec 1992

Self-injection of fish vaccine can cause anaphylaxis.

by Leira HL, Baalsrud KJ

This article in PubMed: www.ncbi.nlm.nih.gov/pubmed/1485168

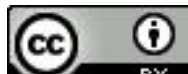




\section{Self-injection of fish vaccine can cause anaphylaxis}

In 1991 three cases of anaphylactic reactions among vaccinators of farmed fish were registered in Norway. The cases developed after repeated self-injection of vaccine against salmon furunculosis.

Aquaculture has been a growing industry along the Norwegian coast during the last decade. Atlantic salmon, which is the main product, is produced mainly for export, at a volume five times that of the second largest producing country, the United Kingdom (1990).

Infectious diseases have been an increasing problem in the industry. Vibriosis (caused by Vibrio anguillarum) and cold-water vibriosis (caused by Vibrio salmonicida) are now controlled by vaccination. The fish is dipped in a bacterial solution or is injected intraperitoneally with the bacteria. No adjuvants have usually been applied with these vaccines.

Another infection, furunculosis, caused by Aeromonas salmonicida subsp salmonicida, was introduced with smolt imported from Scotland in 1985 and is now considered endemic in all major fish-breeding areas along the Norwegian coast. A number of test vaccines based on the causative agent have been tried in Norway since 1987 . So far acceptable protection against the disease seems to depend on the use of injectable vaccines containing adjuvants. The four vaccines used in Norway in 1991 were all based on Formalin ${ }^{\circledR}$-inactivated bacteria and contained aluminum hydroxide, aluminum phosphate, mineral oil, or glycan as the adjuvant. They all contain about $10^{10}$ bacteria per milliliter, and each fish receives a dose of $0.2 \mathrm{ml}$.

In 1991, some 60 million salmon at a weight of approximately $50 \mathrm{~g}$ were vaccinated against furunculosis. The fish are vaccinated as smolts in fresh water hatcheries, prior to sea transfer for the net pen growth period.

The vaccine is injected intraperitoneally after the fish have been anesthetized. The vaccinator holds the fish in one hand, administering the automatic repeating syringe containing the vaccine with the other hand. A skilled vaccinator can inject up to 1500 doses of vaccine per hour. Prototypes for automatic vaccination have been developed, but as yet they are considered too expensive or too slow for routine use.

Following accidental self-injection of vaccine, inflammatory reactions occur in the fingers or the hand and arm holding the fish. More seldom an "influenza" like syndrome with fever, muscle ache, and general malaise occurs, probably caused by endotoxins in the vaccine. Serious anaphylactic reactions (respectively, coma, dyspnea, and confusion) requiring hospitalization have been reported three times. All three persons recovered.

Devices to protect the fingers against accidental selfinjection have been developed, and results so far are promising. However, until fish vaccines can be administered by automatic injection or furunculosis vaccines for dipping or oral administration are developed, self-injection will remain a potentially serious hazard in the aquaculture industry.

\footnotetext{
Håkon L Leira, MD, ${ }^{1}$ Karen $\mathbf{J}$ Baalsrud, DVM ${ }^{2}$

1 Department of Occupational Medicine, University Hospital, N-7006 Trondheim, Norway.

2 National Veterinary Institute, PO BOX 8156 Dep, N-0033 Oslo 1, Norway.
} 